\title{
The Investigation of Euler's Totient Function Preimages
}

\author{
Ruslan Skuratovskii \\ Kiev, MAUP, faculty of computer sciences, Ukraine
}

How to cite this paper: Ruslan Skuratovskii. (2019) The Investigation of Euler's Totient Function Preimages. Journal of Applied Mathematics and Computation, 3(3), 591-598. DOI: $10.26855 /$ jamc.2019.03.001

*Corresponding author: Ruslan Skuratovskii, Kiev, MAUP, faculty of computer sciences, Ukraine. Email: ruslan@unicyb.kiev.ua, ruslcomp@mail.ru.

\begin{abstract}
We propose a lower bound for computing quantity of the inverses of Euler's function. We answer the question about the multiplicity of $m$ in the equation $\varphi(x)=m$ [5]. An analytic expression for exact multiplicity of $m=2^{2 n}+a$, where $a \in N, a<2^{n}$, $\varphi(t)=2^{2 n}+a$ was obtained. A lower bound of inverses number for arbitrary $m$ was found. We make an approach to Sierpinski assertion from new side. New numerical metric was proposed.
\end{abstract}

\section{Keywords}

Euler's totient function, inverses of Euler's function, numerical metric, Fermat Numbers, Chen's Theorem, number of preimages of Euler's totient function, Sierpinski assertion.

\section{Introduction}

The purpose of this work is to study theoretical numerical properties a multivalued function [1,2] which is inversed to Euler's function, show the relevance of the examples.

Subject of study: explore the composition of the function $\varphi(n)$ with itself and the tasks associated with it, it's properties, the number of preimages of the function $\varphi(n)$, behavior of the straight $O A_{n}$, where $A_{n}(n ; \varphi(n))$ and $O(0 ; 0)$ where $n \rightarrow \infty$.

We propose a lower estimation for computing quantity of the inverses of Euler's function. Our approach can be further adapted for computing certain functions of the inverses, such as their quantity, the larger.

Of fundamental importance in the theory of numbers is Euler's totient function $\varphi(n)$. Two famous unsolved problems concern the possible values of the function $A(m)$, the number of solutions of $\varphi(x)=m$, also called the multiplicity of $m$.

Of big importance in the cryptography has number of preimages of Euler's totient function $\varphi(n) ; n=p q$. Because it determines cardinal of secret key space in RSA [8].

\section{Number of values of $\varphi^{-1}(m)$ for special classes of numbers}

We propose a exact formula for computing quantity of the inverses of Euler's function for any number of form $2^{s}$.

An old conjecture of Sierpinski asserts that for every integer $k>2$, there is a number $m$ for which the equation $\varphi(t)=m$ has exactly $k$ solutions the number of solutions $t$ of $\varphi(t)=m$, also called the multiplicity of $m$. In this section we find multiplicity for numbers of form $2^{s}$.

Example 1. The set of preimages for 12 is following: $\phi^{-1}(12)=\{13 ; 21 ; 26 ; 28 ; 36 ; 42\}$. Also we have $\varphi^{-1}(16)=\{32 ; 48 ; 17$; $34 ; 40 ; 60\}, \varphi^{-1}(18)=\{19 ; 27 ; 38 ; 54\}$. We remind, that the number of a form $2^{2^{n}}+1$, where $n$ is not-negative integer, is called Fermat number.

Also the recursive formula for Fermat numbers $[1,3,4,6]$ was used: $F_{n}=F_{0} \ldots F_{n-1}+2$. Useful for the study of the number of prototypes is Lucas's Theorem: each prime divisor of the Fermat number $F_{n}$, where $n>1$, has a form of $k 2^{n+2}+1$.

Lemma 1. If $2^{m}+1$ is prime, then $m=2^{n}$

Proof. We will prove by contradiction. Suppose there exists a number of a form $2^{m}+1$ which is not prime and $m$ is divisible by $p \neq 2$. Since $p$ is prime and it is not 2 , it must be odd. Let $m=p t$, so we can rewrite our number like this: $2^{m}+1=\left(2^{t}\right)^{p}+(1)^{p}=$

$\left(2^{t}+1\right)\left(\left(2^{t}\right)^{p-1}-\ldots+(1)^{p-1}\right)$. Expressions in both brackets are grater than 1 , but our number is supposed to be prime. Contradiction.

We make of use Theorem about mutually coprimality of non-prime Fermat number [6]. 
Theorem 1. Let $n \in N \cup\{0\}$. If $2^{2^{n}}+1$ is not prime, then for any number of the form $2^{2^{n}+a}$, where $a \in N$, a< $2^{n}$, there exists exactly $2^{t}$ natural numbers $m$ such that $\varphi(m)=2^{2^{n+a}}$, where $t$ is amount of prime Fermat numbers, which are less than $2^{2^{n}}+1$.

Proof. Consider a set $\left\{p_{1} ; p_{2} ; \ldots p_{t}\right\}$ of all prime Fermat numbers lesser than $2^{2^{n}}+1$. Let $\varphi(x)=2^{2^{n}+a}$. According to Lemma 1 , $x=2^{s} q_{1} q_{2} \ldots q_{v}$, where $q_{i}$ are different prime Fermat numbers. Since $a<2^{n}$, then $2^{2^{n}+a}<2^{2^{n+1}}$. That means, that $q_{i}<2^{2^{n+1}}+1$, because $\varphi(x)=\varphi\left(2^{s} q_{1} q_{2} \ldots q_{v}\right)=2^{2^{n}+a}<2^{2^{n+1}}$.

We also know that $q_{i} \neq 2^{2^{n}}+1$, because $2^{2^{n}}+1$ is not prime. This yields $q_{i}<2^{2^{n}}+1$. Other words it can be written like this: $\left\{q_{1}\right.$, $\left.q_{2}, \ldots q_{v}\right\} \subseteq\left\{p_{1}, p_{2}, \ldots p_{t}\right\}$. For each $x$ we get, that $\left\{q_{1}, q_{2}, \ldots q_{v}\right\}$ is a subset of the set $\left\{p_{1}, p_{2}, \ldots p_{t}\right\}$. We shall prove, that each subset of the set $M_{t}=\left\{p_{1}, p_{2}, \ldots p_{t}\right\}$ determines such unique $x$ as a unique product of this subset of primes from $M_{t}$, that $x$ with a corresponding multiplier $2^{s}, s \in N \cup\{0\}$ gives us $x=2^{s} t$ such that $\varphi(x)=2^{2^{n}+a}$.

For this goal we need to show, that $\varphi\left(p_{1} \cdot p_{2} \ldots p_{t}\right)<2^{2^{n}+a}$.

Since $\varphi\left(p_{1} \cdot p_{2} \ldots p_{t}\right)$ is Euler's function of a product of prime Fermat numbers, which lesser than $2^{2^{n}}+1$, it is not grater than value of Euler's function of a product of all Fermat numbers, which lesser than $2^{2^{n}}+1$, which lesser than $2^{2^{n}}+1$, which is equal to

$$
\varphi\left(\left(2^{2^{0}}+1\right) \ldots\left(2^{2^{n-1}}+1\right)\right)
$$

That is true, as obvious inequality holds: $\varphi(d) \varphi(d b)$. It is also known, that any two Fermat numbers are coprime [6], so

$$
\varphi\left(\left(2^{2^{0}}+1\right) \ldots\left(2^{2^{n-1}}+1\right)\right)=\varphi\left(2^{2^{0}}+1\right) \ldots \varphi\left(2^{2^{n-1}}+1\right) .
$$

As known, $\varphi(y) \leq y-1$, therefore

$$
\varphi\left(2^{2^{0}}+1\right) \ldots \varphi\left(2^{2^{n-1}}+1\right) \leq\left(2^{2^{0}}+1-1\right) \cdot \ldots \cdot\left(2^{2^{n-1}}+1-1\right)=2^{0} \cdot \ldots \cdot 2^{2^{n-1}}=2^{2^{n}-1} .
$$

It was used the formula of the sum of geometric progression, we have $2^{0}+2^{1}+\ldots+2^{n-1}=2^{n}-1$. Therefore $\left(2^{2^{0}}+1-1\right) \cdot .$. . $\left(2^{2^{n-1}}+1-1\right)=2^{2^{0}+2^{1}+. .+2^{n-1}}=2^{2^{n}-1}$.

Finally,

$$
\varphi\left(p_{1} \cdot p_{2} \ldots p_{t}\right) 2^{2^{n}-1}<2^{2^{n}+a} ;
$$

what was needed. That means, that Euler's function of the product of the elements of any subset of the set $\left\{p_{1}, p_{2}, \ldots p_{t}\right\}$ is lesser than $2^{2^{n}+a}$. Let us take an arbitrary subset of $\left\{p_{1}, p_{2}, \ldots p_{t}\right\}$. Let the elements of this set be $\left\{q_{1}, q_{2}, \ldots q_{v}\right\}$. Consider the expression $\varphi\left(q_{1} \cdot q_{2} \cdot \ldots \cdot q_{v}\right)=2^{w}<2^{2^{n}+a}$. This inequality means, that we can choose such natural number $s$, so $\varphi\left(2^{s} \cdot q_{1} \cdot q_{2} \cdot \ldots \cdot q_{v}\right)$ $=2^{s-1} \cdot 2^{w}=2^{2^{n}+\mathrm{a}}$. In other words, for given subset $\left\{q_{1}, q_{2}, \ldots q_{v}\right\}$, we found such number $x$, that $\varphi(x)=2^{2^{n}+a}$. The last equality means, that each subset defines unique $x$. Therefore, each subset gives us the needed the number $x$ that is always determined by some subset. In other words, the amount of needed numbers is exactly the amount of different possible subsets. As well-known fact, this amount is equal $2^{t}$ for a set of $t$ elements.

Example 2. For a non-prime Fermat number $2^{32}+1$; number of preimages for subsequent numbers of the form $2^{2^{n}+a}$; a $\leq 32-1$; $n \leq 4$ is equal to $2^{32}$.

For generalizing of Theorem 2 it is easy to prove the following statement:

Theorem 2. Let $a \in Z ; 0 \leq a \leq 2^{n}$, then the number of solutions of $\varphi(x)=2^{2^{n}+a}$ is equal to the number of sets $\left\{2^{i_{1}}, \ldots, 2^{i_{k}}\right\}$, such that:

$$
\begin{aligned}
& i_{1}<i_{2}<\ldots<i_{k} \\
& 2^{i_{1}}+2^{i_{2}}+\ldots+2^{i_{k}} \leq 2^{n}+a \\
& 2^{2^{i_{1}}}+1, \ldots, 2^{2^{i_{k}}}+1 \in F_{p r}
\end{aligned}
$$

where $F_{p r}$ is a set of Ferma's prime numbers.

If $2^{2^{n}}+1$ is not prime, then the number of specified sets (including empty set) is equal to $2^{t}$, where $t$ is a number of Ferma's prime numbers smaller then $2^{2^{n}}+1$.

Proof. To construct the necessary preimage $x$ over the set of Ferma's primes with the properties of this Theorem $\varphi(x)=2^{2^{n}+a}$ we proceed as follows: 
1) We choose a combination of this numbers. Let us call it

$$
\left(2^{2^{i_{0}}}+1\right) \ldots\left(2^{2 i_{k-1}}+1\right)
$$

2) Then we should find its total power of 2 that is $2^{i_{1}}+2^{i_{2}}+\ldots+2^{i_{k}}=s$, this power be obtained after calculating the Euler's function from the product $\varphi\left(\left(2^{2 i_{0}}+1\right) \ldots\left(2^{2 i_{k}}+1\right)\right)$ and also satisfies the inequality

$$
s=2^{i_{1}}+2^{i_{2}}+\ldots+2^{i_{k}} \leq 2^{n}+a .
$$

We supplement the received power exponent $\mathrm{s}$ to the necessary $2^{n}+a$ by multiplying the product of

$$
\left(2^{2^{i_{0}}}+1\right) \ldots\left(2^{2^{i_{k}}}+1\right)
$$

on $2^{2^{n}+a-s}$. Thus, the necessary preimage $x$ is constructed.

Property 1. For any number $S$ of the form $p_{1}^{a_{1}} p_{2}^{a_{2}} \ldots p_{k}^{a_{k}}, p_{1}>2$, where $p_{1}, p_{2}, \ldots, p_{k}$ are odd prime numbers, the following equality holds: $\varphi(S)=\varphi(2 S)$ :

Proof. Since 2 and $p_{1}^{a_{1}} p_{2}^{a_{2} \ldots} p_{k}^{a_{k}}, p_{1}>2$ are coprime, then

$$
\varphi\left(2 p_{1}^{a_{1}} p_{2}^{a_{2}} \ldots p_{k}^{a_{k}}\right)=\varphi(2) \varphi\left(p_{1}^{a_{1}} p_{2}^{a_{2}} \ldots p_{k}^{a_{k}}\right)=\varphi\left(p_{1}^{a_{1}} p_{2}^{a_{2}} \ldots p_{k}^{a_{k}}\right) .
$$

Therefore these numbers has the same of Euler's function.

Theorem 3. If $\varphi(m)=2^{n}$, then $m=2^{s} p_{1} p_{2} \ldots p_{x}$, where $p_{i}$ are different Fermat numbers, $s \in N$.

Proof. Firstly, we will prove that $m$ can be divisible by odd prime number $p$, only if $p$ is prime Fermat number. Let's consider an arbitrary prime number $\mathrm{p}$ such that $m: p$. Then $m=p_{1}^{a_{1}} p_{2}^{\mathrm{a}_{2}} \ldots p_{x}^{a_{x}} \cdot p^{a}$, where $a \geq 1$. As Euler's function is multiplicative and $\varphi\left(p^{x}\right)$ $=p^{x-1}(p-1)$, as well-known fact, we can write:

$$
\varphi(m)=\varphi\left(p_{1}^{a_{1}} p_{2}^{a_{2}} \ldots p_{x}^{a_{x}}\right) \cdot \varphi\left(p^{a}\right)=\varphi\left(p_{1}^{a_{1}} p_{2}^{a_{2}} \ldots p_{x}^{a_{x}}\right) \cdot p^{a-1}(p-1) .
$$

Therefore, $\varphi(m) \vdots(p-1)$. If ( $p-1)$ is divisible by odd prime number $q$, then $\varphi(m)$ is also divisible by $q$, which can't be, because $\varphi(m)=2^{n}$. That is why $p-1$ can be divisible only by 2 , which yields $(p-1)=2^{k}$, or $p=2^{k}+1$. According to lemma, $k$ $=2^{n}$, so we can rewrite $p=2^{2^{n}}+1$. In other words, $p$ is Fermat prime number, what was needed.

Secondly, we will prove that $m$ can’t be divisible by $p^{2}$, where $p$ is prime, $p \neq 2$. We will prove by contradiction. If $m$ is divisible by $p^{2}$, then $m=p_{1}^{a_{1}} p_{2}^{a_{2}} \ldots p_{x}^{a_{x}} \cdot p^{a}$, where $a \geq 2$. As we already know,

$$
\varphi(m)=\varphi\left(p_{1}^{a_{1}} p_{2}^{a_{2}} \ldots p_{x}^{a_{x}}\right) \cdot \varphi\left(p^{a}\right)=\varphi\left(p_{1}^{a_{1}} p_{2}^{a_{2}} \ldots p_{x}^{a_{x}}\right) \cdot p^{a-1}(p-1) .
$$

so $\varphi(m) \vdots p$. But $p \neq 2$, while $\varphi(m)=2^{n}$. Contradiction.

\section{Low bound of $\varphi^{-1}(m)$ values for number of general form}

We propose a lower bound for computing quantity of the inverses of Euler's function. Our approach can be further adapted for computing certain functions of the inverses, such as their quantity, the larger.

Let $M_{k}$ be a set of first $k$ consecutive primes. We will say, that the number is decomposed over a set $M_{k}$, if in its canonical decomposition there are only numbers from $M_{k}$. Let $x_{1}, \ldots, x_{n+2}$ be such numbers, that $\varphi\left(x_{1}\right)=\varphi\left(x_{2}\right)=\ldots=\varphi\left(x_{n+2}\right)$, and at the same time all prime factors of the canonical decomposition belong to the set $M_{n}=\left\{p_{0}, \ldots, p_{n}\right\}$, where $p_{0}=2$ and $p_{i}$ are all consecutive prime numbers. Let for any natural number $n$, we have $Q_{n}=\left(p_{0}-1\right)\left(p_{1}-1\right) \ldots\left(p_{n-1}-1\right)\left(p_{n}-1\right)$, where $p_{i}$ is $i$-th odd prime number, where $i \in N$ and $p_{0}=2$. Example: $p_{1}=3, p_{2}=5, p_{3}=7$, then $Q_{3}=\left(p_{0}-1\right)\left(p_{1}-1\right)\left(p_{2}-1\right)\left(p_{3}-1\right)=$ $(3-1)(5-1)(7-1)=48$.

Let $M_{k}$ be a set of $k$ consequent first prime numbers. The following statement about estimation of preimages number is true.

Theorem 4. For any natural number $n$ there are such various natural numbers $x_{1}, x_{2}, \ldots, x_{n}, x_{n+1}, x_{n+2}$, that

$$
\varphi\left(x_{1}\right)=\varphi\left(x_{2}\right)=\ldots=\varphi\left(x_{n+2}\right)=Q_{n},
$$


where every number $x_{i}$ contains in its canonical decomposition [6] only $p_{i}$ from $M_{n}$, and

$$
x_{n+2}=p_{0} p_{1} \ldots p_{n-1} p_{n}
$$

holds.

Proof. We prove it by mathematical induction.

Base case: given $n=1$, then $P 1=\left(p_{1}-1\right)=2$ has at least three prototypes. This statement is true, because $\varphi(3)=\varphi(4)=\varphi(6)=2$. Base case is proved.

Step case: if for $n=k$ it holds, we will prove, that for $n=k+1$ it holds too. By the assumption we have, that for natural number $n$ were found such various natural $x_{1}, x_{2}, \ldots, x_{k+1}, x_{k+2}$, that

$$
\varphi\left(x_{1}\right)=\varphi\left(x_{2}\right)=\ldots=\varphi\left(x_{k+1}\right)=\varphi\left(x_{k+2}\right)=Q_{k}=Q,
$$

where $Q_{k+1}=p_{0}^{\beta_{1}} p_{1}^{\beta_{2}} \ldots p_{k}^{\beta_{x}}$,

$$
x_{k+1}=p_{1} p_{2} \ldots p_{k-1} p_{k}, x_{k+2}=p_{0} p_{1} p_{2} \ldots p_{k-1} p_{k} .
$$

Let us make induction transition. Prove, that for $n=k+1$ exist such various natural $y_{1}, y_{2}, \ldots, y_{k+2} ; y_{k+3}$, for which holds:

$$
\varphi\left(y_{1}\right)=\varphi\left(y_{2}\right)=\ldots=\varphi\left(y_{k+2}\right)=\varphi\left(y_{k+3}\right)=Q_{k+1},
$$

each of which has a canonical decomposition over $M k \cup p_{k+3}$. Clear, that $\varphi\left(p_{k+3}\right)$ has a canonical decomposition into elements of $M_{k}$. Therefore, it can be presented as

$$
\varphi\left(p_{k+3}\right)=p_{0}^{\beta_{1}} p_{1}^{\beta_{2}} \ldots p_{k}^{\beta_{x}} .
$$

Let's construct new numbers $y_{1}, y_{2}, \ldots, y_{k+1}, y_{k+2}, y_{k+3}$ such that:

$$
y_{1}=x_{1} p_{k+3}, y_{2}=x_{2} p_{k+3}, \ldots, y_{k+1}=x_{k+1} p_{k+3}, y_{k+2}=x_{k+2} p_{k+3}
$$

In this case, the value of the Euler function is $Q_{k+1}=p_{0}^{\beta_{1}} p_{1}^{\beta_{2}} \ldots p_{k}^{\beta_{x}}$. Let us show, that all $y_{1}, \ldots, y_{k+2}, y_{k+3}$ are different.

Since numbers $x_{1}, x_{2}, \ldots, x_{k+1}, x_{k+2}$ from (1) have different canonical decomposi-tions, so the decompositions of numbers $y_{1}, y_{2}, \ldots, y_{k+1}, y_{k+2}$ over $M_{k}$ are different too, but they all have a new factor $\mathrm{pk}+1$, but do not decompose over Mk. A last one $\mathrm{yk}+3$ also decomposes over Mk and does not contain a factor $p_{k+1}$. But value $Q_{k+1}=p_{0}^{\beta_{1}} p_{1}^{\beta_{2}} \ldots p_{n}^{\beta_{n}}$ does not contain $p_{k+1}$ in the decomposition, so there is at least one number $y_{k+3}$ with decomposition over $M_{k}$, such, that $\varphi\left(y_{k+3}\right)=Q_{k+1}$ holds.

Since $Q_{k+1}>Q_{k}$, then a new preimage $y_{k+3}$ does not coincide with any of the numbers $y_{1}, y_{2}, \ldots, y_{k+1}, y_{k+2}$ which give the value of Euler's function equal $Q_{k}$.

Moreover such $y_{k+3}$ can be not unique number that can be constructed over $M_{k}$ such, that $\varphi\left(y_{k+3}\right)=Q_{k+1}$. Consequently beyond $y_{1}, y_{2}, \ldots, y_{k+1}, y_{k+2}$, which decomposed over $M_{k+1}$, we have at least one new $y_{k+3}$, which can be decomposed over $M_{k}$ in product of primes. Thus $Q_{k+1}$ has at least $k+3$ different preimages.

We propose method of constructing of such preimages set.

Let $p_{0}=2, p_{1}=3, p_{2}=5, \ldots, p_{n}$ be consecutive prime numbers. Note, that $\varphi\left(p_{0} p_{1}, \ldots ; p_{n}\right)=\left(p_{1}-1\right)\left(p_{0}-1\right) \ldots\left(p_{n}-1\right)$. Let's construct some other numbers $x_{0}, \ldots, x_{n}$, for which

$$
\varphi\left(x_{0}\right)=\varphi\left(x_{1}\right)=\ldots=\varphi\left(x_{n}\right)=\varphi\left(p_{0}, p_{1}, \ldots, p_{n}\right)=\left(p_{0}-1\right)\left(p_{1}-1\right) \ldots\left(p_{n}-1\right) .
$$

Namely, let

$$
\begin{aligned}
& x_{0}=\left(p_{0}-1\right) p_{0}, p_{1}, \ldots, p_{n} \\
& x_{1}=p_{0}\left(p_{1}-1\right) p_{2}, \ldots, p_{n} \\
& \ldots \\
& x_{n}=p_{0} p_{1}, \ldots, p_{n-1}\left(p_{n-1}-1\right)
\end{aligned}
$$


Now let's prove, that $\varphi\left(p_{0} p_{1} \ldots p_{k-1}\left(p_{k}-1\right) p_{k+1} \ldots p_{n}\right)=\left(p_{0}-1\right)\left(p_{1}-1\right) \ldots\left(p_{n}-1\right)$ for every $k=0,1, \ldots, n$. Obviously, $p_{0} \ldots p_{k-1}\left(p_{k}-1\right)$ and $p_{k+1} \ldots p_{n}$ are coprime, so $\varphi\left(x_{k}\right)=\varphi\left(p_{0} p_{1} \ldots p_{k-1}\left(p_{k}-1\right)\right) \times \varphi\left(p_{k+1} \ldots p_{n}\right)=\varphi\left(p_{0} p_{1} \ldots p_{k-1}\left(p_{k}-1\right)\right) \times\left(p_{k+1}-1\right) \ldots\left(p_{k}-1\right)$ That is, we have to prove the equality $\varphi\left(p_{0} p_{1} \ldots p_{k-1}\left(p_{k}-1\right)\right)=\left(p_{0}-1\right)\left(p_{1}-1\right) \ldots\left(p_{k}-1\right)$.

Since only $p_{0} p_{1}, \ldots, p_{k-1}$ are the prime numbers, which are not more than $\left(p_{k}-1\right)$, we have $p_{k}-1=\alpha_{0} \alpha_{1}, \ldots, \alpha_{k-1}$ for some nonnegative integer $\alpha_{0} \alpha_{1}, \ldots, \alpha_{k-1}$.

By direct calculation we obtain

$$
\begin{aligned}
& \varphi\left(p_{0} p_{1} \ldots p_{k-1}\left(p_{k}-1\right)\right)=\varphi\left(p_{0}^{\alpha_{0}+1} p_{1}^{\alpha_{1}+1} \ldots p_{k-1}^{\alpha_{k-1}+1}\right)= \\
& \left(p_{0}-1\right) \ldots\left(p_{k-1}-1\right) p_{0}^{\left(\alpha_{0}+1\right)-1} \ldots p_{k-1}^{\left(\alpha_{k-1}+1\right)-1}= \\
& =\left(p_{0}-1\right)\left(p_{1}-1\right) \ldots\left(p_{k-1}-1\right) p_{0}^{\alpha_{0}} \ldots p_{k-1}^{\alpha_{k-1}}=\left(p_{0}-1\right)\left(p_{1}-1\right) \ldots\left(p_{k-1}-1\right)\left(p_{k}-1\right)
\end{aligned}
$$

Also we may subtract 1 from more than one $p_{k}$, if $\left(p_{k}-1\right)$ has the decomposition into prime factors, which does not contain some $p_{j}(j<k)$. For example, $\varphi\left(p_{0} p_{1} p_{2} p_{3}\right)=\varphi(2 \times 3 \times 5 \times 7)=48$. Except $(2-1) \times 3 \times 5 \times 7,2 \times(3-1) \times 5 \times 7,2 \times 3 \times(5-1) \times$ 7 and $2 \times 3 \times 5 \times(7-1)$, we may take, for example, $2 \times(3-1)(5-1) \times 7$, because $(3-1)=2$ and $(5-1)=2^{2}$. Hence $\varphi(2 \times(3$ $-1)(5-1))=(2-1)(3-1)(5-1)$ by the same arguments, as for $p_{0} p_{1} \ldots p_{k-1}\left(p_{k}-1\right) p_{k+1}, \ldots, p_{n}$. So, we may construct at most $2^{n}$ products of the form $p_{0} q_{1}, \ldots q_{n}$, where $q_{k}=p_{k}$. Also $\left(p_{0}-1\right) p_{1}, \ldots, p_{n}$ fits for the requirement $\varphi\left(p_{0}-1\right) p_{1}, \ldots, p_{n}=\left(p_{0}-1\right) \ldots$ $\left(p_{n}-1\right)$, so we have at most $2^{n}+1$ numbers, which give us the same meaning of $\varphi$, as $p_{0}, \ldots, p_{n}$. Note, that it is not necessarily the complete set of such numbers $x$, for which $\varphi(x)=p_{0} p_{1}, \ldots, p_{n}$, but it is the set, which may be obtained by the given by us scheme.

An old conjecture of Sierpinski asserts that for every integer $k>2$, there is a number $\mathrm{m}$ for which the equation $\varphi(t)=m$ has exactly $k$ solutions the number of solutions $t$ of $\varphi(t)=m$, also called the multiplicity of $m$. In this section we We find multiplicity for numbers of form $m=2^{2} 3^{x}, m=2^{k} 3^{n}$. denote by $\eta p(x)$ the $p$-adic power exponent of number $m$ viz such minimal number of $n$ that $m=t^{n} a$, where $a$ is divisor of unit in the ring $Z_{p}$.

Theorem 5. There exists 3 classes of possible preimages of $m$ which satisfy $\varphi(t)=2^{k} 3^{n}$ :

1. If $t=2^{y} 3^{x}$, then $y \in\{1, \ldots, k+1\}, x \in N \cup\{0\}$.

2. If $t=2^{y} 3^{z} p_{1}$ and the following conditions holds:

$$
\left\{\begin{array}{l}
(\mathrm{x}-1)+1+\eta_{2}\left(\varphi\left(p_{i}\right)\right)=k, \\
\mathrm{y}-1+\eta_{3}\left(\varphi\left(p_{1}\right)\right)=n .
\end{array}\right.
$$

3. If $t=2^{x} 3^{y} \cdot \prod_{i=1}^{L} p_{i}=1$ where $p_{i} \in P$ such that $p_{i}=2^{v_{i 1}} 3^{v_{i 2}}+1$ in particular $p_{i}$ can be prime Fermat number. For such numbers the following conditions hold:

$$
x+\sum_{i=1}^{L} \eta_{2}\left(\varphi\left(p_{i}\right)\right)=k \text { and } y-1+\sum_{i=1}^{L} \eta_{3}\left(\varphi\left(p_{i}\right)\right)=n
$$

Proof. Proof is carried out by direct verification. So the resulting power of two in $m$, from the item 3 , is the following $(x-1)+$ $1+\sum_{i=1}^{L} \eta_{2}\left(\varphi\left(p_{i}\right)\right)=x+\sum_{i=1}^{L} \eta_{2}\left(p_{i}\right)=k$. Also resulting power of 3 is such $y-1+\sum_{i=1}^{L} \eta_{3}\left(\varphi\left(p_{i}\right)\right)=n$. On account of the set of primes in item 3 consists of number of form

$$
p_{i}=2^{u_{i}} 3^{v_{i}}+1
$$

let quantity of such number is $L$ and numbers in form of prime Fermat number $p_{i}=2^{2^{i}}+1$ let sum of such number is $F$, then $k-x-F$ can be splited in sum of number of primes of first form and second form. If we now $F$ for concrete $m$, then we express multiplicity of m as $C_{k-F+L-x-1}^{L-1}$.

Recall that quantity of splits of form $v_{1}+v_{2}+\ldots+v_{L}=n-y+1$ can be counted by the formula $C_{n-y+1+L-1}^{L-1}$. Consider the canonical decomposition of factor $\prod_{i=1}^{L} p_{i}$ from item 3. The expression of the exact number of methods of splits of the numbers $n$ and $k$ (which appear in item 3 ) is reduced to finding the number of pairs of such vectors $\left(u_{L}, \ldots, u_{L}\right)$ and $\left(v_{1}, \ldots, v_{m}\right)$ that 
$\sum_{i=1}^{L} u_{i}=k$ and $\sum_{i=1}^{L} v_{i}=n$ with respect of the columns of form

$$
\left(\begin{array}{l}
u_{i} \\
v_{i}
\end{array}\right)
$$

must be pairwise different. In this case, in the matrix of degrees 2 and 3 in the preimages, which has the form

$$
\left(\begin{array}{llll}
v_{0} & v_{1} & \ldots & v_{L} \\
u_{0} & u_{1} & \ldots & u_{L}
\end{array}\right)
$$

where $u_{0}=x+F, v_{0}=y-1$. Then the formula is calculated

$$
C_{k+L-F-y-1}^{L-1}
$$

will give us the exact number of partitions. Also, when calculating the number of combinations (3) using the formula, we assume that the matrices are the same, which are obtained from each other by rearranging the columns even if these columns are different. Other words, the number of splits $C_{k+L-F-y-1}^{L-1}$ considers different partitions where the parts can be rearranged, but in our case the parts are terms and therefore nothing changes from the permutation. Therefore, it necessary to divide by factorial $L$ ! Thus, we obtain

$$
\frac{C_{k+L-F-y-1}^{L-1}}{L !}
$$

Where $F$ are numbers in form of prime Fermat number $p_{i}=2^{2^{i}}+1$ let sum of such number is $F$. This formula counts distributions by all number of form $p_{i}=2^{u_{i}} 3^{v_{i}}+1$ but not all of them are prime, consequently we obtain an upper bound.

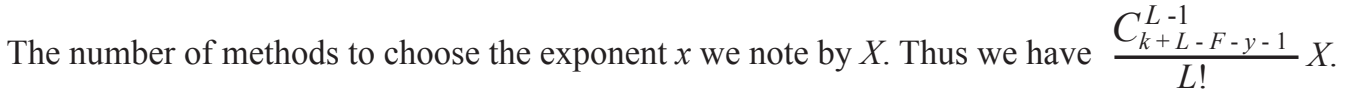

Also the formula (3) distinguish splits with a same values of $\left(u_{i}, v_{i}\right)$ and $\left(u_{j}, v_{j}\right)$. numbers. Then we have to apply exception inclusion formula. According to this formula we have to subtract a number of intersections of sets with the same columns of powers $\left(u_{i}, v_{i}\right)$.

The exact number of partitions is estimated from above by the exception inclusion formula, if we stop at the even intersection and below at the odd one. We need to evaluate

$$
I=\left|U-\bigcup_{\substack{i, j=1 \\ i<j}}^{L} M_{i j}\right|
$$

where $U$ is the set of all distributions of $k-F-y$ into $L$ terms, we denote it by $I$. Let us present the equations of powers form item 3) in form of two equations

$$
\left\{\begin{array}{l}
x_{0}+x_{1}+\ldots+x_{L}=k \\
y_{0}+y_{2}+\ldots+y_{L}=n
\end{array}\right.
$$

where $x_{0}=x+F, x_{i}=u_{i}, y_{1}=v_{i}$ are defined in the item 3), we count coinciding of columns (pairs) of form $x_{i}=x_{j}, y_{i}=y_{j}$.

Let $U$ is set of all splits of form (5) that starts from $i=1$ 


$$
\left|U-\bigcup_{\substack{i, j=1 \\ i<j}}^{L} M_{i j}\right| \leq|U|-\sum_{i=1}^{L-1} \sum_{j=i+1}^{L}\left|M_{i j}\right|+\sum_{i=1}^{L-1} \sum_{\substack{j_{1}=i_{1}+1 \\\left(i_{1}, j_{1}\right) \neq\left(i_{2}, j_{2}\right)}}^{L} \sum_{i_{2}=1}^{L-1} \sum_{j_{2}=i_{2}+1}\left|M_{i_{1} j_{1}} \cap M_{i_{2} j_{2}}\right|-\ldots
$$

For each columns (pairs) of power $(i, j):\left|M_{i j}\right|=\sum_{k=0}^{\left[\frac{k}{2}\right]} \sum_{l=0}^{\left[\frac{n}{2}\right]} C_{x-2 k+m-3}^{m-3} C_{y-2 l+m-3}^{m-3}$, where $k, l$ are values of powers of 2 and 3 in pairs where these values $k, l$ are coincided. Therefore to count exact value we should to substitute cardinal of intersection. Further we should count power set of intersections $\left|M_{i j} \cap M_{p q}\right|$ by the similar principle. The prove is completed.

Corollary 6. The upper bound of the preimages of $m$ for fixed $m$ in $\varphi(t)=m$ is

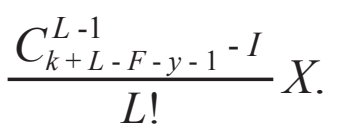

This bound is upper because formula (4) count all numbers of form $p_{i}=2^{u_{i}} 3^{v_{i}}+1$ but not all of them are prime.

Example 3. Number of the preimages for $m=2$ is 3 because $\varphi(3)=\varphi(4)=\varphi(6)=2$ this preimages belongs to first case. Also $\varphi(5)=\varphi(12)=\varphi(8)=\varphi(10)=22$ thispreimages belongs to 3$)$ and 1) cases.

The calculation shows that cardinal of preimages set depends of $h=2^{\eta_{2}(m)}, \eta_{2}(m)>1$ and $\eta_{2}(m), \eta_{3}(m)>1$ for investigation this dependence we propose new metric, which we dedicate to the Academic Pratzyvyty M.

$$
p(h, g)=\left|\ln \frac{h(1-g)}{g(1-h)}\right|, h, g>1
$$

where in general case instead 1 it can be $c=$ const and $h, g>c>0$ (or $c>h, g>0$ ). We shortly prove inequality of triangle. If all logarithm not negative then $\ln \left(\frac{x(1-y)}{y(1-x)}\right) \leq \ln \left(\frac{x(1-z)}{z(1-x)}\right)+\ln \left(\frac{z(1-y)}{y(1-z)}\right)$ since logarithm is monotonic and continual function, then, after adding the logarithms in left part, we proceed to $\frac{x(1-y)}{y(1-x)} \leq \frac{x(1-z) z(1-y)}{z(1-x) y(1-z)}=\frac{x(1-y)}{y(1-x)}$. Therefore we prove the equality in this case. Other one difficult case if one of logarithms, for instance $\ln \left(\frac{z(1-y)}{y(1-z)}\right)$, is negative then $\left|\ln \left(\frac{x(1-y)}{y(1-x)}\right)\right|=$ $\left|\ln \left(\frac{x(1-z)}{z(1-x)} \frac{z(1-y)}{y(1-z)}\right)\right|=\left|\ln \left(\frac{x(1-z)}{z(1-x)}\right)+\ln \left(\frac{z(1-y)}{y(1-z)}\right)\right| \leq\left|\ln \left(\frac{x(1-z)}{z(1-x)}\right)\right|+\left|\ln \left(\frac{z(1-y)}{y(1-z)}\right)\right|$. Thus, inequality holds. Property $p(g, h)$ $=(h, g)$ is true due to modulo and property of logarithm.

We will describe this dependence of $g=2^{\eta_{2}(m)}, h=3^{\eta_{3}(m)}$ in the next work.

\section{Conclusion}

The analytic expression for exact multiplicity of inverses for $m=2^{2^{n}+a}$, where $a \in N, a<2^{n}$ and $\varphi(t)=m$ was obtained. As it turned out, it depends on the number of prime numbers Fermat. Upper and lower bounds for arbitrary number $n$ were obtained by us. The method of constructing of preimages set for obtained by us lower bound was proposed by us.

\section{References}

[1] Křížek, M., Luca, F., \& Somer, L. (2001). 17 lectures on Fermat numbers: from number theory to geometry. Springer.

[2] Coleman, R. (2009). On the image of euler's totient function. Mathematics.

[3] Ruslan Skuratovskii (2018). The investigation of Euler's totient function preim-ages. In Sixth International Conference on Analytic Number Theory and Spatial Tessellations. Voronoy Conference (pp. 37-39)

[4] Skuratovskii, R. (2017). INVOLUTIVE IRREDUCIBLE GENERATING SETS AND STRUCTURE OF SYLOW 2-SUBGROUPS OF ALTERNATING GROUPS. Romai journal, 13(1).

[5] Ford, K. (1999). The number of solutions of $\varphi(x)=m$. Annals of Mathematics, 150(1), 283-311. 
[6] Vinogradov, I. M. (2016). Elements of number theory. Courier Dover Publications.

[7] Skuratovskii, R. V. (2017). Structure and minimal generating sets of Sylow 2-subgroups of alternating groups. Source: https://arxiv. org/abs/1702.05784 v2.

[8] Bakhtiari, M., \& Maarof, M. A. (2012). Serious security weakness in RSA cryptosystem. International Journal of Computer Science Issues (IJCSI), 9(1), 175.

[9] Alekseyev, M. A. (2016). Computing the inverses, their power sums, and extrema for Euler's totient and other multiplicative functions. Journal of Integer Sequences, 19(2), 3. 